\title{
Editorial
}

\section{Logros y retos en la formación especializada en medicina y enfermería del trabajo tras 10 años del programa oficial de la especialidad}

\section{Achievements and challenges in occupational medicine and nursing specialization after 10 years of the official specialization program}

\section{Victoria Ureña Villardiel', Jerónimo Maqueda Blasco²}

1. Subdirección General de Investigación en Terapia Celular y Medicina Regenerativa. Instituto de Salud Carlos III. Madrid. España.

2. Comisión Nacional de la Especialidad de Medicina del Trabajo. Instituto Nacional de Seguridad e Higiene en el Trabajo. Madrid. España.

Recibido: 19-11-15

Aceptado: 02-12-15

\section{Correspondencia}

Jerónimo Maqueda Blasco

Presidente de la Comisión Nacional de la Especialidad de Medicina del Trabajo.

Instituto Nacional de Seguridad e Higiene en el Trabajo. Madrid. España.

Correo electrónico: jmaqueda@insht.meyss.es

En 2006, comenzaba a impartirse el nuevo programa oficial de la Especialidad de Medicina del Trabajo, aprobado en la Orden SCO/1526/2005 y con ello se abría una nueva etapa en la historia de la Medicina del Trabajo en nuestro país, historia que había arrancado en 1946, con la creación de la Escuela Nacional de Medicina del Trabajo.

Esta nueva etapa suponía hermanar esta especialidad con el resto de especialidades médicas, fortaleciendo la competencia clínica del especialista sin abandonar la competencia preventiva, que es y debe seguir siendo el "core" de la Medicina del Trabajo: debemos gestionar la salud para no vernos obligados a gestionar la enfermedad y la incapacidad.

En estos diez año hemos asistido a un fenómeno paradójico, por un lado la madurez de las estructuras docentes representadas por las Unidades Docentes de Medicina del Trabajo y Multiprofesionales y de los Centros Formativos de Referencia, dentro de los que debemos dirigir una especial atención a la Escuela Nacional de Medicina del Trabajo del Instituto de Salud Carlos III, que como Institución ha tenido el acierto de trabajar por un proyecto de Curso Superior de Medicina del Trabajo abierto a la construcción junto con los Jefes de Estudios de la Unidades Docentes y Residentes. Esta convergencia en un Proyecto común ha permitido generar un espacio de innovación y progreso cuyo resultado ha sido una formación MIR de excelencia, como así lo evalúa el propio residente.

Una de las principales brechas que separaban la Medicina del Trabajo de otras especialidades médicas ha sido la escasa producción científica de sus residentes, en este sentido se han producido importantes avances. El convencimiento de la Escuela Nacional de Medicina del Trabajo de que una especialidad se prestigia científicamente y se acredita socialmente en la medida que es capaz de generar conocimiento entorno a los problemas de la Sociedad, ha impulsado un subprograma MIR de formación en investigación, en cual el residente adquiere habilidades en la identificación de problemas de investigación, la formulación de hipótesis y objetivos, la identificación y síntesis de la evidencia, la redacción y presentación científica y el manejo de las herramientas básicas en el tratamiento de datos. 
Este programa ha permitido que el MIR R1 de Medicina del Trabajo destaque, hoy en día, en el manejo del conocimiento científico, marcando una innovación en la formación especializada que debería ser seguida por otras especialidades médicas y recogida en el futuro programa de troncalidad.

Sin embargo, todos los que estamos comprometidos con la formación médica sabemos que la formación especializada no agota el proceso de formación. Las instituciones públicas tienen, frente a la Sociedad, la obligación de promover un marco adecuado para la sostenibilidad de la competencia del médico en ejercicio. Esto implica la movilización permanente del conocimiento a través de herramientas de formación y de comunicación científica accesibles. El aprovechamiento de las TICS para la formación médica continuada y la comunicación científica son críticas para mantener una equidad en el acceso del profesional sanitario a su derecho (de acuerdo a la Ley de Ordenación de las Profesiones Sanitarias) a una formación continua.

Hoy en día los especialistas en Medicina del Trabajo tenemos la oportunidad de acceder a una oferta de e-formación jerarquizada en base a necesidades percibidas por el profesional sanitario en ejercicio (tanto medicina como enfermería) y el acceso libre y a texto completo de la producción científica que se genera entorno a la salud Laboral, en un proyecto editorial en castellano con una mayor indexación en base de datos internacionales como la Revista Medicina y Seguridad en el Trabajo.

Los esfuerzos realizados por el Instituto de Salud Carlos III a través de la Escuela Nacional de Medicina del Trabajo, hace que los profesionales de Medicina y Enfermería del Trabajo disfruten de mayores oportunidades de acceso a la formación continua con respecto a otras profesiones de prevención de riesgos laborales y en esa misma dirección dentro de las especialidades de medicina y enfermería sean de las pocas que dispone de un centro formativo de referencia que garantiza la adecuación de la praxis al progreso y los cambios de la sociedad productiva.

Pero el asentamiento en los logros está reñido con los avances, por lo que se deben abordar nuevos retos en Medicina y Enfermería del Trabajo tanto en formación como en divulgación científica. Algunos de ellos de carácter inmediato como la formación MIR en el marco de la troncalidad, la formación EIR (que debe abordarse sin más dilaciones), el diseño de itinerarios de diplomas de acreditación avanzada en el marco de la formación médica continuada, la internacionalización de la actividad formativa mediante la traslación de programas formativos de éxito a terceros países, una mejor y más transparente gestión editorial de la Revista Medicina y Seguridad del Trabajo y su impulso a la indexación en PubMed, logrando una mayor proyección y visibilidad y por tanto influencia de la producción científica de nuestro país a nivel internacional.

Estos 10 años de avances en formación médica y divulgación científica en Medicina y Enfermería del Trabajo deben abrir una etapa de nuevos desarrollos hacia un proceso de confluencia y agregación de esfuerzos y recursos del conjunto de administraciones públicas que intervienen en los aspectos sanitarios de la prevención de riesgos laborales, en una iniciativa común en Medicina del Trabajo que, enmarcada en la Sociedad del Conocimiento, la Comunicación y la Innovación, dé respuesta a las necesidades de salud de nuestro tejido productivo y de sus actores. 